\title{
Effects of Electrode Relocation on Bioelectrical Impedance Measurements on Potato Tissue
}

\author{
Albert Lozano-Nieto
}

\begin{abstract}
The measurement of bioelectrical impedance is a non-destructive technique that is being used to assess the quality of fruits and plants. Applications of this technique assume that changes in the values of bioelectrical impedance are only due to changes in the tissues under study. This paper evaluates the bioelectrical impedance changes associated to changing the location of the measurement electrodes using an electrode fixture that keeps their distance constant. Due to the short period of time in which these measurements take place, the tissue should have not changed and therefore the measured impedance values should remain constant. This research reports changes in the values of low-frequency resistance as high as $\mathbf{1 5}$ $\%$, changes in the values of high-frequency resistance as high as $39 \%$ and changes in the Cole-Cole frequency as high as $16 \%$. Being these changes in the same order of magnitude, or even larger than changes in bioelectrical impedance reported in the literature for practical applications of the technique, one needs to exercise extreme caution at the time of interpreting similar results in the practical applications of this technique and extracting conclusions.
\end{abstract}

Keywords - bioelectrical impedance, electrode relocation, Cole-Cole frequency, resistance, and reactance.

\section{INTRODUCTION}

The measurement of electrical impedance in biological tissues, commonly known as bioelectrical impedance measurements or impedance spectroscopy, was first developed to study a number of physiological events in humans such as shifts in body fluids during dialysis [1], the relationship between respiration and cardiovascular events [2], the assessment of body composition [3], and other clinical applications that benefit from a safe, non-invasive, and inexpensive technique [4].

More recently, bioelectrical impedance measurements have been used to assess parameters associated to the quality of fruits and plants. These include the study of correlation between changes in bioelectrical impedance values against weight changes due to their ripening during an 8-day period [5] and changes in bioelectrical impedance fruit associated to changes in their mechanical parameters such as their firmness as an alternative to using the traditional techniques which are destructive [6]. Additional applications are the use of bioelectrical impedance un robotic arms to selectively plucking mango fruits from their trees [7], and the assessment of ripening of kiwifruit [8]. On a larger time, scale, bioelectrical impedance has been used to estimate the quality of nectarines at harvest, 3 and 8 weeks after cold storage [9]. A somewhat related approach to the traditional bioelectrical impedance method is to use the dielectric properties of the fruit for which its quality needs to be characterized such as bananas [10] or Garut citrus [11] by inserting their flesh as the dielectric in a capacitor. This information is used develop electrical models of these fruits with parameters that become dependent with the amount of ripening. Bioelectrical impedance is also used to monitor the dehydration process by forced air of potato tissue for its conservation. Dehydration destroys the cellular membrane which in turn changes the frequency behavior of the potato tissue [12]. The quality of eggplant pulp subjected to drying as well as to freezingthawing cycles can also be estimated with the use of bioelectrical impedance using the same approach [13]. Preventing low-quality apples to reach the market after excessive storage time [14] and avoiding bruised apples to also reach the market [15] are also based on studying their bioelectrical impedance characteristics. Additional applications of the bioelectrical impedance technique are its use to differentiate between 'pressed' and 'pulpy' fruits [16], evaluating how live plants acclimate to coldness [17], and the safety assessment of other types of foods [18].

These applications require repeating bioelectrical impedance measurements through the duration of the experiment. Moreover, they assume that the only contribution to bioelectrical impedance changes rests upon changes on the tissue under examination. However, previous research on using bioelectrical impedance measurements to characterize physiological parameters on humans discovered that small differences in the repositioning of the measuring electrodes results on a relatively significant contribution to bioelectrical impedance changes [19]. These changes can mask the underlying physiological changes that need to be measured. This paper evaluates the variability of short-time bioelectrical impedance values on the potato tissues ('Solanum tuberosum') measured at different tissue locations in a very short time frame. Because these measurements at the different locations are taken in less than one hour in total, we can assume that the electrical characteristics of the tissue have not changed. Further, because these measurements are taken using a test fixture that maintains the separation between electrodes constant, these bioelectrical impedance measurements are not affected by changes in the distance between electrodes and therefore will remain constant among the different testing locations. 


\section{METHODS}

\section{A. Bioelectrical Impedance Measurement Probe}

Bioelectrical impedance was measured on a whole potato ('Solanum tuberosum') with intact skin using an Instek Precision LCR meter LCR-6300 which has a measurement range from $10 \mathrm{~Hz}$ to $300 \mathrm{kHz}$. These measurements were performed using its four-wire Test Leads probe $L C R-06 A$ and the needle electrode probe shown in Figure 1 in order to keep the distance between electrodes constants. The measurements were performed in seven different locations on the top of the object under test which are named L1 through L7 in this paper. The direction of the electrode probe was kept constant to avoid potential effects from anisotropy in the tissue. The distance between adjacent test locations was between 1.5 and $2.0 \mathrm{~cm}$. The probe was fully inserted in the tissue in order to keep the contact area fixed for all measurements.

Preliminary measurements on a similar sample found the optimal frequency range for bioelectrical impedance measurements was in the frequency range from $600 \mathrm{~Hz}$ to 100 $\mathrm{kHz}$. Within this frequency range, the Instek instrument measured bioelectrical impedance at 150 frequencies equally spaced, averaging 5 different measurements. The measurements at each location were performed consecutively (L1 through L7), lasted 5 minutes, and the time interval between the end of measurements at one location and the beginning of measurements at the next

location was less than 30 seconds. This short measurement time frame ensures that there are no changes in the tissue under measurement.

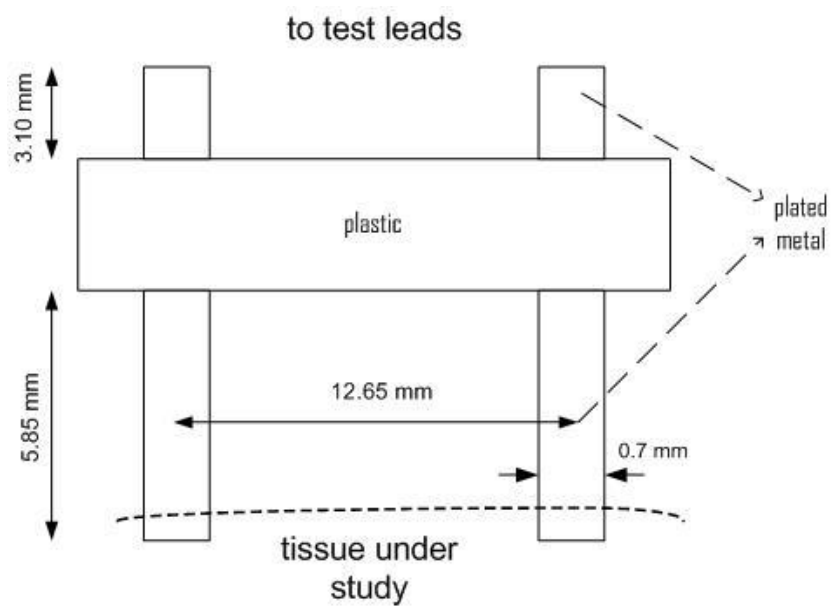

Fig. 1. Electrode test probe for the measurement of bioelectrical impedance.

The electrode probe shown in Figure 1 was built from the plated connector headers used in electronic printed circuit boards which ensures good electrical conductivity due to their platting and therefore minimizes electrode impedance. Bioelectrical impedance from the tissue was measured using the 4-wire, 2 electrode technique. Although several papers in the literature report using surface electrodes [5], [6], [15], [16], it is important to note that surface electrodes have been designed to monitor physiological events on humans. Therefore, the chemical composition of their electrolyte has been optimized to convert the ionic current generated by human muscles into the electrical current to be recorded by electronic instruments and may not have the optimal characteristics for applications in plants and fruits Furthermore, while the surface area of these electrodes is small compared to the size of a human body and thus assuring homogeneous current distribution, this does not hold true when these surface electrodes are used on plants or fruits. Therefore, the short distance between electrodes generates a non-homogenous current distribution that invalidate the underlying assumptions used for bioelectrical impedance measurements. Given all these considerations, the probe shown in Figure 1 was selected for this experiment.

\section{B. Methods}

Being impedance a complex number, it is typically represented by its real and imaginary components. These are also affected by frequency of the electrical signal used to measure them. Using the simplistic, yet useful model for a tissue shown in Figure 2, it is possible to estimate the frequency behavior of the tissue under study. In this model, $\mathrm{Re}$ represents the resistance of the extracellular fluids, $\mathrm{Ri}$ represents the resistance of the intracellular fluid and $\mathrm{C}$ represents the cellular membrane. This cellular membrane acts like capacitor which impedance can be modelled as $Z_{c}=$ $\frac{1}{s^{\alpha} C}$ with $s$ being the complex frequency, $\mathrm{C}$ the value of the capacitance and $\alpha$ a parameter that is dependent on specific tissues.

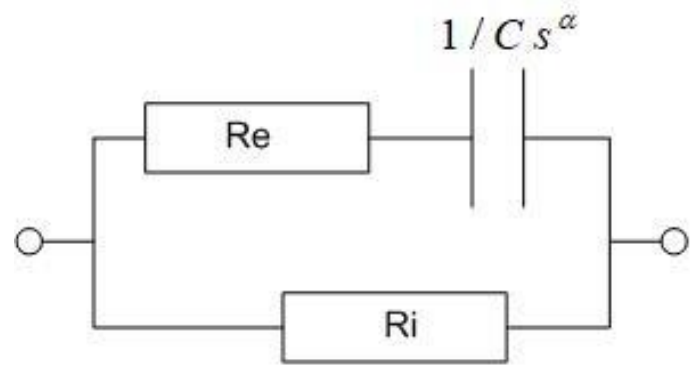

Fig. 2. Electrode test probe for the measurement of bioelectrical impedance.

At very low frequencies, the capacitor has an extremely large reactance which results in the cell membrane presenting a strong opposition to the flow of current. Therefore, the current flows mostly through the extracellular medium. The measured impedance then becomes the impedance of the extracellular tissue. Conversely, at very high frequencies, the reactance of the capacitor decreases which causes the capacitor to act like a short circuit. This allows the free flow of current through both extra- and intra cellular tissues. With this, the value of bioelectrical impedance measured is the parallel combination of the intra and extracellular components. Given that the extracellular component is already know from the measurement at very low frequencies, it is now possible to estimate the value of the intracellular component of the tissue. In these two extreme values of frequencies, the imaginary part of the impedance is very small and therefore bioelectrical impedance can be represented by just its real part (resistance). At intermediate frequencies, there is a portion of the current flowing solely through the extracellular component while a different portion of the current flow through its intracellular components. This results in bioelectrical impedance having both, a real part (resistance) and an imaginary part (reactance). 
Fig. 3 displays the resistance and reactance for the tissue under study over the range of frequencies that was used (600 $\mathrm{Hz}$ to $100 \mathrm{kHz}$ ). It is important to note that, because the tissue has a capacitive behavior resulting in a negative reactance, the plot typically displays the negative part of the imaginary part of impedance. Figure 3 shows how the real part of the impedance decreases as the frequency of the current increases. Furthermore, the increase in frequency results in more current flowing through the cellular membrane which decreases the value of the overall resistance. Figure 3 also shows how the value of the imaginary part of the impedance is minimal at the two frequency extremes. By using the concept of Cole-Cole curve, it is possible to extrapolate that at zero frequency -direct current- the imaginary part will be zero as all well as at infinity frequency will be also zero.
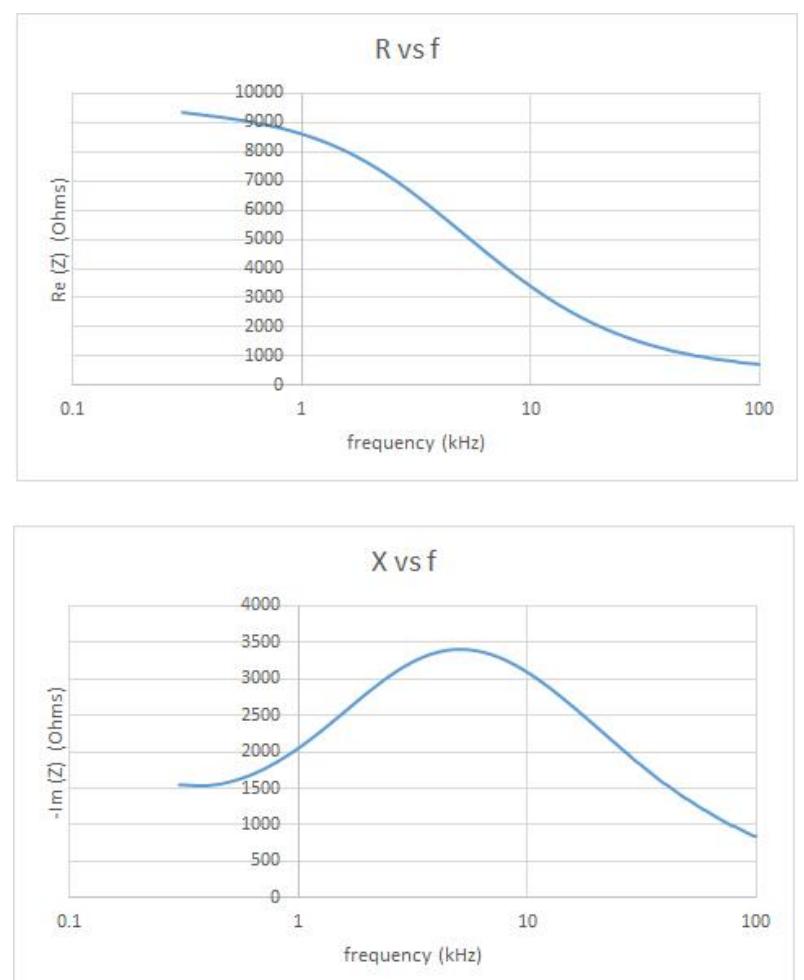

Fig. 3. (a) Frequency dependence of the real part of the bioelectrical impedance of the tissue under study; (b) Frequency dependence of the imaginary part of the bioelectrical impedance of the tissue under study.

A frequency of special interest known as the Cole-Cole frequency-, is the frequency at which the reactance (imaginary part of the impedance) for the tissue under study becomes maximal. Because it determines the boundary between the imaginary part of the impedance increasing versus decreasing, knowing the value of this frequency is critical at the time of determining the optimal range of frequencies for bioelectrical impedance measurements. This range is selected such as the Cole-Cole frequency is located around the middle point of this frequency range. Displaying the imaginary part of the bioelectrical impedance against its real part is known as the Cole-Cole plot which is typically used to summarize graphically bioelectrical impedance data.

Fig. 4 shows the Cole-Cole plot for the tissue under study which frequency behavior was represented in Fig. 3. Because the actual frequency range is limited between $600 \mathrm{~Hz}$ and 100 $\mathrm{kHz}$, the plot does not reach a zero value of the imaginary part of the impedance. Instead, the dashed lines show the extrapolation of Ro and $\mathrm{R}_{\infty}$. Ro represents the extrapolated value of the resistance at $\mathrm{DC}$ and $\operatorname{Max}|\mathrm{X}|$ represents the maximum of the absolute value of the reactance in the tissue Furthermore, this figure also shows how at the Cole-Cole frequency (fc) the imaginary part of bioelectrical impedance reaches its maximal value.

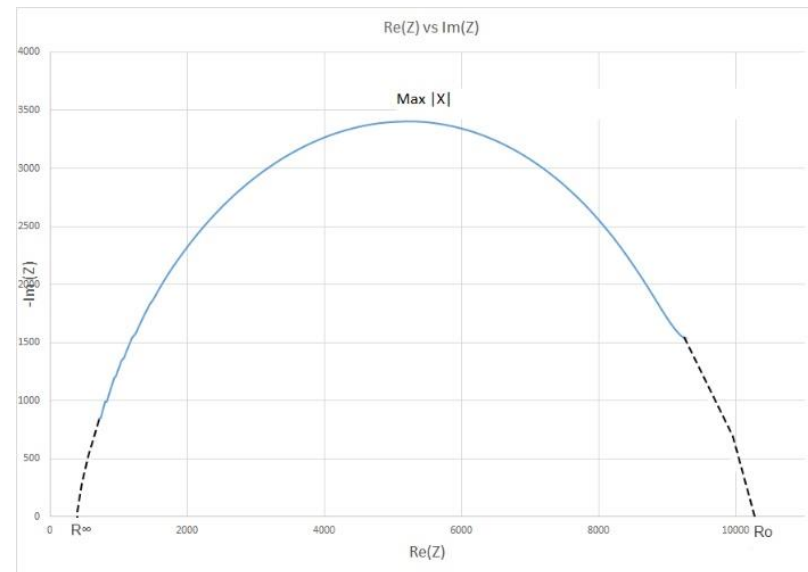

Fig. 4. Representation of the imaginary part of bioelectrical impedance against its real part.

\section{RESULTS}

Table I summarizes the extrapolated values of Ro, $\mathrm{R}_{\infty}$ and fc at each one of the seven different locations. These results show that, although there were no changes in the tissue itself, the values of bioelectrical impedance represented through these 3 parameters exhibited a strong variation. Ro represents the resistance at $600 \mathrm{~Hz}, \mathrm{R}_{\infty}$ represents the frequency at 100 $\mathrm{kHz}$ and $\mathrm{fc}$ represents the frequency at which the imaginary part of the complex impedance value is the highest in absolute value which corresponds to the top of the Cole-Cole circle.

TABLE I: IMPEDANCE VALUES AND CRITICAL FREQUENCY

\begin{tabular}{cccc}
\hline \hline $\begin{array}{c}\text { Measurement } \\
\text { Location }\end{array}$ & Ro $(\Omega)$ & $\mathrm{R}_{\infty}(\Omega)$ & $\mathrm{fc}(\mathrm{kHz})$ \\
\hline L1 & 8,694 & 791 & 5.3 \\
L2 & 8,978 & 978 & 5.0 \\
L3 & 8,903 & 682 & 5.3 \\
L4 & 9,299 & 923 & 5.6 \\
L5 & 7,998 & 753 & 5.9 \\
L6 & 8,321 & 740 & 5.9 \\
L7 & 8,393 & 673 & 5.9 \\
\hline \hline
\end{tabular}

Table II summarizes the maximal changes in the values of Ro, $R_{\infty}$ and $\mathrm{fc}$, showing that they are not negligible. In fact, these exceed one standard deviation although they are contained within three standard deviations. The change in absolute magnitude in bioelectrical impedance becomes more severe at the lowest frequencies although the percentual change in bioelectrical impedance has a stronger contribution at the highest frequencies.

TABLE II: SUMMARY OF IMPEDANCE AND CRITICAL FREQUENCY CHANGES

\begin{tabular}{c|c|c|c|c}
\hline \hline Parameter & $\begin{array}{c}\Delta \max \\
(\text { absolute })\end{array}$ & $\Delta \max (\%)$ & Mean & $\begin{array}{c}\text { Standard } \\
\text { deviation }\end{array}$ \\
\hline Ro & $1301 \Omega$ & $15 \%$ & $8655 \Omega$ & $412 \Omega$ \\
$\mathrm{R} \infty$ & $314 \Omega$ & $39 \%$ & $793 \Omega$ & $111 \Omega$ \\
$\mathrm{fc}$ & $0.9 \mathrm{kHz}$ & $16 \%$ & $5.56 \mathrm{kHz}$ & $0.337 \mathrm{kHz}$ \\
\hline \hline
\end{tabular}


Fig. 5 depicts that the two locations that showed the larger changes for Ro (L4 and L5) occurred in the middle of the measurements. This implies that the time in which the tests were performed does not have an influence in these changes.

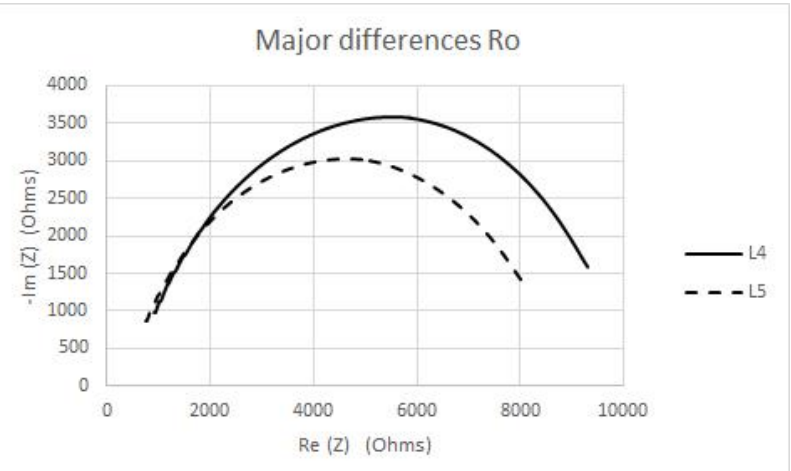

Fig. 5. Maximal differences on the values of Ro among different test locations

Additionally, the two locations in which R $\infty$ had the largest variations (L4 and L7) also occurred in the middle stages if the experiment as it is shown in Fig. 6. Once again, this shows that the time in which the tests were performed does not have an influence in these changes.

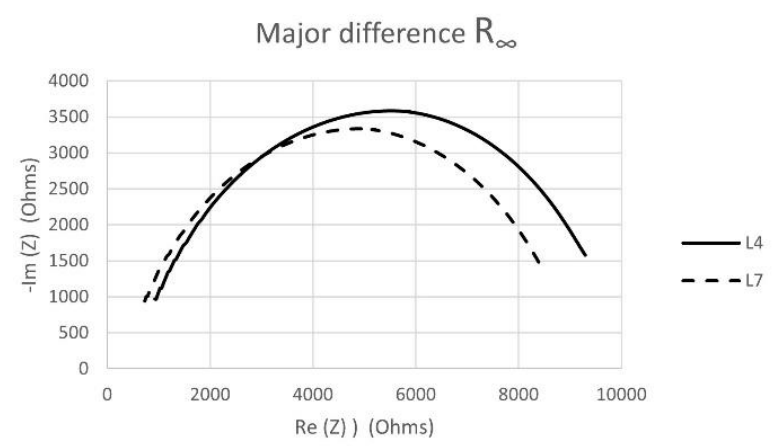

Fig. 6. Maximal differences on the values of $R_{\infty}$ at different test locations.

The Cole-Cole plots from Fig. 5 and 6 provide clear evidence that measuring bioelectrical impedance at different locations, on the same tissue, while keeping the same general orientation for the current probe within the tissue, results in considerable variations on the measured values of bioelectrical impedance. These variations contradict the generalized assumption that bioelectrical impedance measurements remain constant when measured at different locations within the tissue. In order to better characterize the observed changes in bioimpedance values, it is important to evaluate how these changes in impedance vary with the measurement frequency. This is shown Fig. 7 which displays the maximal relative differences as error curves in the real and imaginary parts of the bioelectrical impedance measurements taken at the seven testing locations (L1 through L7).

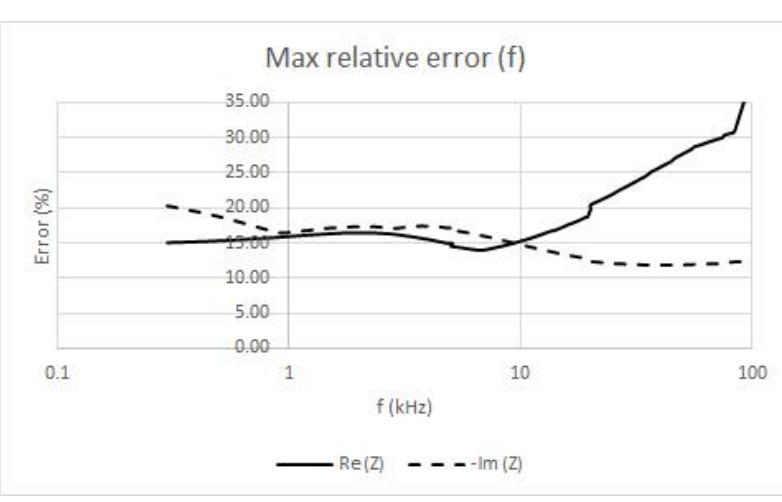

Fig. 7. Maximum relative error in real and imaginary parts against frequency.

Fig. 7 indicates the existence of a relatively constant variation for both, real and imaginary parts of bioelectrical impedance for the frequencies between $600 \mathrm{~Hz}$ and $10 \mathrm{kHz}$. At frequencies higher than $10 \mathrm{kHz}$, however, there is a marked increase in relative error for the real part of bioelectrical impedance which implies a stronger variability in measured bioelectrical impedance values. These results agree with the data summarized in Table 1, showing a variation of approximately $15 \%$ at low frequencies, and raising up to beyond $30 \%$ for higher frequencies. The imaginary part of bioelectrical impedance shows less variability compared to the real part, as it is contained in a narrower band. Finally, it is important to note the existence of a frequency range ( $1 \mathrm{kHz}$ to $10 \mathrm{kHz}$ in this experiment) in which both real and imaginary parts of impedance show the lowest variability.

\section{DISCUSSION}

The results of this experiment show the existence of clear and marked changes on bioelectrical impedance values when these are measured at different locations even during a short period of time which prevents physiological changes in the tissue. Moreover, as the measuring locations are close to each other and the measuring probe was positioned in the same general direction, it is possible to conclude that the observed variations in the values of bioelectrical impedance were not due to the experimental setup. These result raise concerns about the reliability of bioelectrical impedance data for assessing the quality of fruits and plants when measurements are taken at different locations or at different times as these conditions require the repositioning of measurement electrodes. Therefore, it becomes critical to identify and separate which bioelectrical impedance changes are due to changes in the fruits or plants and which ones are due to the repositioning of the electrodes.

As reported in the introduction, bioelectrical impedance measurements have been used by to study changes in fruit and plant tissues over a period of time. It is then necessary to ensure that these changes exceed the variability of bioelectrical impedance values reported in Table II. Otherwise, the conclusions from evaluating the data from their experiments may be misleading. The results of this research show that repeated bioelectrical impedance measurements taken on the same tissue, even within a very short time frame, are affected by the location of the measurement electrodes. These findings contradict the 
underlying assumption that bioelectrical impedance data is only affected by the state of the tissue itself. While these experiments were carried out using a fixed fixture to keep the distance among electrodes constant, it is possible to envision that variability in bioelectrical impedance will be more pronounced when using surface electrodes. In this case, one must account for additional changes due to not keeping the distance between electrodes constants. Therefore, any applications of the bioelectrical impedance technique focusing on extracting conclusions based on the data measured, must evaluate its results with extreme care. Being able to differentiate the origin of these changes thus becomes critical to validate the use of the bioelectrical impedance technique in practical applications. Given these results, it is not possible to discard that actual changes in the tissue under study may be masked by the variability arising from electrode repositioning in which even small positional changes result on larger variations of bioelectrical impedance data. Furthermore, while both, low- and high-frequency resistance values exhibit changes as a result changing the location of the measurement electrodes, the percentual change in the value of high-frequency resistance is greater than the percentual changes for low-frequency resistance values. However, due to the lower baseline value for high-frequency resistance, these changes tend to be masked by the larger values of lowfrequency resistance. Furthermore, the frequency at which bioelectrical impedance is measured has a direct effect on the variability of its results. Prior to using bioelectrical impedance in practical applications, it may be necessary to analyze this dependence to select the optimal frequency range, paying especial consideration to the high frequency resistance.

\section{CONCLUSION}

From the investigation carried out, it is evident that the measurements of bioelectrical impedance on a potato tissue show variability as these are measured at different locations. It is therefore possible to extrapolate those bioelectrical measurements on other plants and fruits will also be affected by this variability. Therefore, researchers in this field should consider this variability at the time of extracting conclusions on their data.

This research also found that the the imaginary part of the bioelectrical impedance presents lower variability compared to the real part of the bioelectrical impedance over the whole frequency range. This fact suggests that future applications based on measuring bioelectrical impedance characteristics on fruits and plant need to consider its imaginary part which has been traditionally not used. Further research is needed to investigate localized anisotropy in plant or fruit tissues as a potential reason for data variability. Finally, conclusions extracted from using bioelectrical impedance values must exercise extreme caution when correlating changes in bioelectrical impedance data with physiological changes in the tissues under study.

\section{FUNDING}

This research was partially funded by a Research and Development Grant, Engineering Technology and Commonwealth Engineering, College of Engineering, The Pennsylvania State University.

\section{CONFLICT OF INTEREST}

Author declares that they do not have any conflict of interest.

\section{REFERENCES}

[1] Zhu F, Schneditz D, Kaufman A, Levin N. Estimation of body fluid changes during peritoneal dialysis by segmental bioimpedance analysis. Kidney International. 2000; 57(1):299-306.

[2] Turner A, Lozano-Nieto A, Bouffard M. Effects of ventilation on reproducibility of bioimpedance spectroscopy fluid ratio measures. $J$. Breath Res. 2008;2(2):026005.

[3] Lozano-Nieto A. Human Body Composition Determination. J. Clinical Eng. 1998;23(6):416-422.

[4] Lozano-Nieto A. Clinical Applications of Bioelectrical Impedance Measurements. J. Clinical Eng. 2000;25(4):211-218.

[5] Chowdhury A, Singh P, Bera T, Ghoshal D, Chakraborty B. Electrical impedance spectroscopic study of mandarin orange during ripening. $J$. Food Meas. Characterization. 2017;11(4):1654-1664.

[6] Figueiredo Neto A, Cárdenas Olivier N, Rabelo Cordeiro E, Pequeno de Oliveira H. Determination of mango ripening degree by electrical impedance spectroscopy. Comp. Elect. in Agriculture. 2017;143:222 226.

[7] Rehman M, Abu Izneid B, Abdullah M, Arshad M. Assessment of quality of fruits using impedance spectroscopy. Int. J. Food Science \& Tech. 2011;46(6):1303-1309.

[8] Bauchot A, Harker F, Arnold W. The use of electrical impedance spectroscopy to assess the physiological condition of kiwifruit. Postharvest Biology Tech. 2000;18(1):9-18.

[9] Harker F, Dunlop J. Electrical impedance studies of nectarines during coolstorage and fruit ripening. Postharvest Biology Tech. 1994;4(12):125-134.

[10] Soltani M, Alimardani R, Omid M. Evaluating banana ripening status from measuring dielectric properties. J. Food Eng. 2011;105(4):625631.

[11] Juansah J, Budiastra IW, Dahlan K, Seminar KB. Electrical behavior of Garut citrus fruits during ripening. Change in resistance and capacitance models of internal fruits. Int. J. Hist. Eng.Tech. 2012;12(4): 1-8.

[12] Ando Y, Mizutani K, Wakatsuki N. Electrical impedance analysis of potato tissues during drying. J. Food Eng. 2014;121:24-31.

[13] Wu L, Ogawa Y, Tagawa A. Electrical impedance spectroscopy analysis of eggplant pulp and effects of drying and freezing-thawing treatments on its impedance characteristics. J. Food Eng. 2008;87(2):274-280.

[14] Watanabe T, Ando Y, Orikasa T, Kasai S, Shiina T. Electrical impedance estimation for apple fruit tissues during storage using ColeCole plots J. Food Eng. 2018;221:29-34.

[15] Jackson P, Harker F. Apple Bruise Detection by Electrical Impedance Measurement. Hort Sc. 2000;35(1):104-107.

[16] Vozary E, Benko P. Non-destructive determination of impedance spectrum of fruit flesh under the skin. J. Phys.: Conf. Ser. 2010;(224), 012142 .

[17] Stout D, Hall J, McLaughlin N. In vivo plant impedance measurements and characterization of membrane electrical properties: The influence of cold acclimation. Cryobiology. 1987;24(2):148-162.

[18] Pliquett U. Bioimpedance: A review for food processing. Food Eng. Rev.: 2010;(2), 74-94

[19] Lozano A, Rosell J, Pallas-Areny R. Errors in prolonged electrical impedance measurements due to electrode repositioning and postural changes. Phys.Meas.1995;(16):121-130. 